\title{
Interaction potential and transport properties of $\mathrm{NeO}^{+}$
}

\author{
Daniel M. Danailov and Larry A. Viehland \\ Science Division, Chatham University, Pittsburgh, Pennsylvania 15206 USA \\ Rainer Johnsen \\ Department of Physics and Astronomy, University of Pittsburgh, Pittsburgh, Pennsylvania 15260 USA \\ Timothy G. Wright \\ School of Chemistry, University of Nottingham, University Park NG7 2RD, United Kingdom \\ Edmond P. F. Lee \\ School of Chemistry, University of Southampton, Highfield, Southampton SO17 1BJ, United Kingdom
}

(Received 4 June 2007; accepted 27 June 2007; published online 22 August 2007)

\begin{abstract}
The results of both experimental and theoretical studies of the mobility of $\mathrm{O}^{+}$in $\mathrm{Ne}$ are reported. Errors in the experiments have been carefully assessed, allowing the obtained data to serve as stringent tests of the $a b$ initio potentials. These potentials were calculated using the RCCSD(T) method, employing basis sets of quintuple- $\zeta$ quality. Curves were calculated for the lowest ${ }^{4} \Sigma^{-}$state [arising from $\mathrm{O}^{+}\left({ }^{4} S\right)$ interacting with $\mathrm{Ne}$ ] and for the ${ }^{2} \Pi$ state [arising from $\mathrm{O}^{+}\left({ }^{2} D\right)$ interacting with $\mathrm{Ne}$. Then, the effects of spin-orbit coupling were incorporated by using the Breit-Pauli operator. The resulting ground state $(\Omega=3 / 2)$ of $\mathrm{NeO}^{+}$gives mobility values in good agreement with experiment at all field strengths. Values of spectroscopic quantities for the ground electronic state of $\mathrm{NeO}^{+}$are also presented. (C) 2007 American Institute of Physics. [DOI: 10.1063/1.2762221]
\end{abstract}

\section{INTRODUCTION}

Because of the importance of $\mathrm{O}^{+}$reactions in the upper atmosphere and because of the need to analyze drift-tube data on ion-molecule reaction rates, the transport properties of atomic oxygen cations moving through the rare gases have been of considerable interest ${ }^{1-9}$ since 1970. In those studies, experimental uncertainties in the measured mobilities were deemed acceptable even when they were as large as $10 \%$, since the data were needed only to provide energy scales for reaction-rate studies. Data of better accuracy are needed to test ion-neutral interaction potentials with measured mobilities.

The transport and reaction properties of $\mathrm{O}^{+}$ions were the focus of an important trio of papers ${ }^{10-12}$ in 1977. It was shown that the non-Maxwellian speed distribution of atomic ions poses no serious problems in the interpretation of the dependence on translational energy of ion-neutral rate coefficients that are measured in drift tubes or in flowingafterglow systems containing a drift section. The papers show that rate coefficients obtained at low gas temperature and high values of $E / n_{0}$, the ratio of the electric field strength to the gas number density, are largely equivalent to values that would be measured in the low-field limit at a high gas temperature, provided that effects of internal (e.g., vibrational or rotational) excitation of the reagent molecules can either be ignored or can be measured separately by other techniques. Explicit expressions were provided ${ }^{11}$ for easily determining the equivalent high gas temperature.

For the past few years, we have been generating accurate potential energy curves for a large number of systems involving atomic ions and atoms. Our work with closed-shell cations was described in the introduction of a paper ${ }^{13}$ concerning an open-shell anion $\left(\mathrm{O}^{-}\right)$moving through $\mathrm{He}, \mathrm{Ne}$, and Ar. In the latter cases, aug-cc-pV5Z basis sets were employed, together with the full counterpoise correction, to generate potential energy curves for the ${ }^{2} \Sigma^{+}$and ${ }^{2} \Pi$ states that arise from the "hole" in the $\mathrm{O}^{-} 2 p$ shell. We also considered the interaction of these states via the spin-orbit interaction, which yields the ${ }^{2} \Sigma_{1 / 2}^{+},{ }^{2} \Pi_{1 / 2}$, and ${ }^{2} \Pi_{3 / 2}$ states. By comparing calculated transport properties with experimental ones, we were able to deduce that the spin-orbit curves were the ones that were important for describing the interaction of $\mathrm{O}^{-}$with the rare gases. Similarly, careful treatment has recently been reported for $\mathrm{S}^{-}$in $\mathrm{He},{ }^{14}$ although the large error bars on the experimental data precluded definitive decisions in this case. We have also considered some other open-shell systems, where the spin-orbit interaction is not important, owing to the electronic states involved: $\mathrm{Hg}^{+}$and $\mathrm{Cd}^{+}$ions, ${ }^{15}$ as well as $\mathrm{Tl}^{+}$ions, ${ }^{16}$ in all of the rare gases.

Recently, ${ }^{17}$ we reported new experimental measurements for the mobility of $\mathrm{O}^{+}$ions in $\mathrm{He}$ gas at $300 \mathrm{~K}$. The accuracy of these new values was estimated as $\pm 2.5 \%$, which allowed them to serve as a stringent test of a new ab initio potential that we calculated using the RCCSD $(\mathrm{T})$ method and reported in the same paper. We employed the aug-cc-pV5Z basis set with the counterpoise correction and took into account coupling between the ${ }^{4} \Sigma_{3 / 2}^{-}$and the ${ }^{2} \Pi_{3 / 2}$ spin-orbit states, arising from the interaction of $\mathrm{He}$ with $\mathrm{O}+\left({ }^{4} S\right)$ and $\mathrm{O}^{+}\left({ }^{2} D\right)$, respectively. Since the diabatic states cross, one effect of the spin-orbit interaction is to lead to an avoided crossing, and hence to a change in the ion mobility. The purpose of the present work is to make a similar study of the $\mathrm{NeO}^{+}$system. 


\section{EXPERIMENTAL DETAILS}

The Pittsburgh selected ion drift apparatus (SIDA) was first described in $1982 ;^{8}$ a short description can also be found in the book by Mason and McDaniel. ${ }^{18}$ The SIDA consists of an ion drift tube, a pulsed electron-impact ion source, and two differentially pumped quadrupole mass filters, the "injection mass filter" and the "analyzer mass filter." In the present work, only the mobility of the ground state of $\mathrm{O}^{+},{ }^{4} \mathrm{~S}$, was desired. Hence, voltage settings in the ion source were adjusted to produce predominantly $\mathrm{O}^{+}\left({ }^{4} S\right)$ ions and to minimize production of $\mathrm{O}^{+}\left({ }^{2} D\right)$ ions that are easily distinguishable by their lower mobility.

We typically injected $\mathrm{O}^{+}\left({ }^{4} S\right)$ ions derived from $\mathrm{O}_{2}$ into the drift section of the SIDA with kinetic energies from 20 to $60 \mathrm{eV}$. In order to test if injection of ions with excess energy caused objectionable "injection effects" of the kind observed in our studies of $\mathrm{O}^{+}$mobilities in helium, ${ }^{17}$ we conducted a series of measurements in which we varied the injection energy and carefully measured the arrival time of the ions. In contrast to the case of the light gas helium, no significant dependence of the arrival time on injection energy was found when oxygen ions were injected into neon. This result was expected, since the momentum transfer cross sections with $\mathrm{Ne}$ are larger than those with $\mathrm{He}$, and the ion/atom mass ratio is more favorable for thermalization. Our conclusion that injection effects were unimportant is supported by the lack of unphysical density dependences of the ion mobilities at low pressures.

All measurements were performed at room temperature $(300 \pm 2 \mathrm{~K})$. The minor temperature fluctuations were taken into account in converting pressures to gas densities. Mobilities were inferred from the average of the arrival-time distribution of $\mathrm{O}^{+}$ions. In practice, the arrival-time distributions were recorded by a multichannel analyzer and a simple algorithm was used to determine the average times. The observed transit times were corrected by subtracting the short time intervals that ions spent traversing the two mass filters and for electronic delay times.

Additional systematic errors may be caused by inaccuracies of the pressure gauge. Calibration measurements, in which we compared mobilities measured in the present apparatus to the very accurately known (uncertainties of $\pm 1 \%$ ) mobility of atomic neon ions in neon, ${ }^{19}$ indicated that our measured mobilities were systematically too small by $6 \%-8 \%$. It was thought previously ${ }^{17}$ that the pressure gauge (a capacitance manometer) gave incorrect readings, but a recent comparison of the gauge to a freshly calibrated gauge of higher accuracy showed that the original gauge was accurate to better than $1 \%$.

Since the SIDA was primarily designed for measurements of ion-neutral reactions, it has some design features that are not optimal for precise measurements of mobilities. For instance, while the electric field is very uniform (better than $1 \%$ ) in the main drift section of the tube (comprising about $86 \%$ of the total length of $35.66 \mathrm{~cm}$ ), the need to inject the ions from the vacuum chamber in the injection mass filter into the drift section, and subsequently into the vacuum chamber of the analyzer mass filter, requires additional elec- trodes to shield against the potentials of the vacuum enclosures. The drift field in these regions is thus not uniform.

Since the gas pressure and all other quantities that enter into the mobility measurements (for instance, voltage and time measurements) are very precise, it was suspected that the systematic error was caused by a slower drift motion in the regions close to the entrance and exit of the drift tube. Test measurements were made, in which the change of the transit times of ions through the tube was recorded when the potentials between the electrodes near the entrance or those near the exit were doubled compared to their normal value, while keeping the potentials of all other electrodes the same. The results showed that the average drift velocity through the exit region (length $=2.54 \mathrm{~cm}$ ) was very close to that in the main section, but that the average drift velocity in the entrance region (length $=2.64 \mathrm{~cm}$ ) was only about $50 \%$ of that in the main section.

It may seem surprising that the entrance region causes a greater error than the very similar exit region. The reason seems to be that the potentials of the electrodes in the entrance region are far above the ground potential of the vacuum walls, while those in the exit region are near ground potential and are thus less sensitive to imperfect shielding against the vacuum walls. The delay of the ions in the entrance region reduces the ion drift velocity, when averaged over the entire drift length, by a factor of 1.07 and this factor was adopted to correct the measured mobility values. There were some indications that the needed correction factor might be slightly higher at higher $E / n_{0}$, perhaps 1.08 , but such small effects were ignored.

The same factor of 1.07 was applied in our previous study ${ }^{17}$ of $\mathrm{O}^{+}$in $\mathrm{He}$ to account for a putative calibration error of the pressure gauge. We now know that the pressure gauge was accurate, but there is no need to revise the conclusions about the accuracy of the interaction potential. The reason is that the data points given in Figs. 1 and 2 of that paper should have been plotted at $7 \%$ larger values of $E / n_{0}$ with the original mobilities, rather than at $7 \%$ smaller mobilities with the original $E / n_{0}$. The agreement between theory and experiment is only marginally affected by this revision; it improves slightly at $E / n_{0}$ values above the mobility maximum but becomes slightly worse at lower $E / n_{0}$. We have, however, replaced the values in the gaseous ion database maintained at Chatham University ${ }^{20}$ with values in which the raw $E / n_{0}$ values were corrected rather than the pressures and raw mobilities.

Figure 1 shows the standard ion mobilities of $\mathrm{O}^{+}$in $\mathrm{Ne}$ measured here (and corrected as just described) at six different pressures. Over most of the $E / n_{0}$ range, the scatter in the present data appears to be largely random, but the data set at 0.96 Torr seems to be subjected to a systematic error. It is possible that the values were affected by small parasitic charges on the guard rings of the drift tube, since such effects would be more pronounced when the drift voltage is very low, as was the case here. This explanation was suggested by our observation $^{17}$ that mobilities measured subsequent to the occurrence of an accidental gaseous discharge tended to be too low by several percent, even after several hours of recov- 


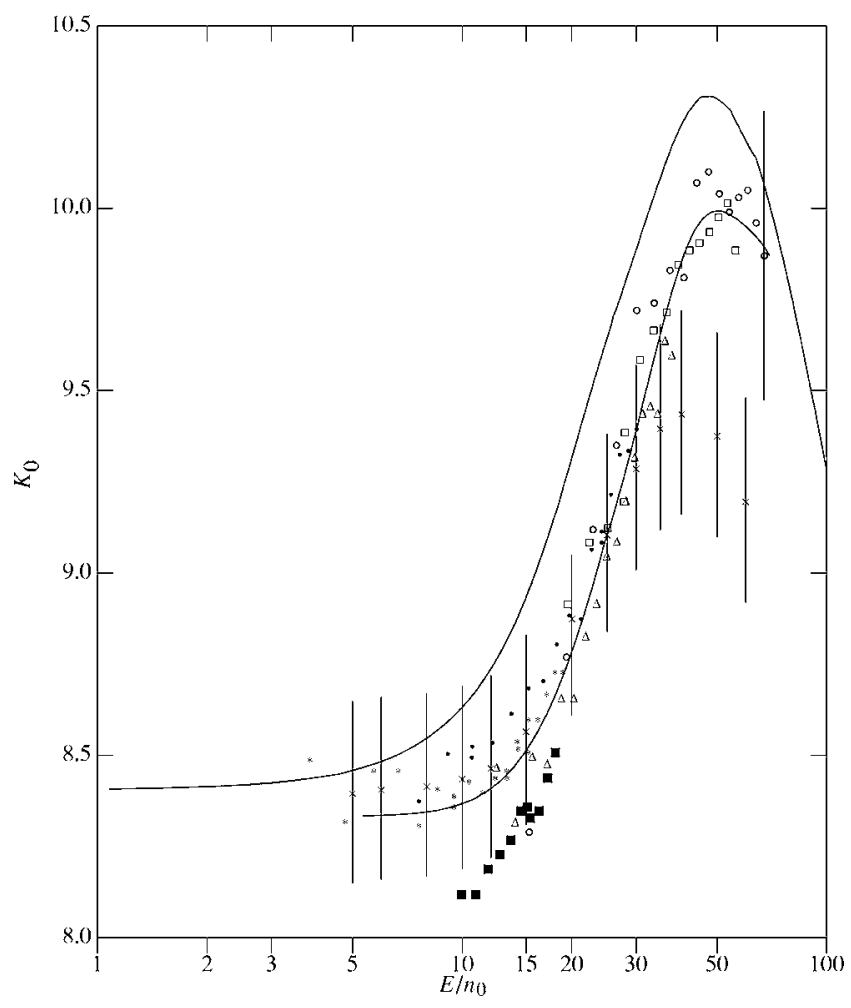

FIG. 1. Experimental values of the standard mobility, $K_{0}$ in $\mathrm{cm}^{2} / \mathrm{V} \mathrm{s}$, as a function of $E / n_{0}$, in Td, for $\mathrm{O}^{+}$ions in Ne at $300 \mathrm{~K}$. The 1982 values of Ref. 8 are represented by the times symbol $(\times)$, with $3 \%$ error bars as estimated by the experimenters. The present values at gas pressures of $0.25,0.30,0.55$, 0.57, 0.90, and 0.96 Torr are represented by circles, squares, deltas, bullets, stars, and boxes, respectively. To avoid clutter, only one of the present values has an attached error bar of $4 \%$. The lower curve represents the mixed fit of the present data, as described in Sec. II. The upper curve represents the values calculated from the present $a b$ intio potential energy function, as described in Sec. III.

ery from the event. Since we cannot be certain that this was the true cause, we chose to include the data set at 0.96 Torr along with the others.

A fit to the present experimental data was constructed in two ways. We first fit the data (using commercial software) to the so-called "log-normal" curve,

$$
K_{0}=K_{0}(0)+b \exp \left\{-0.5\left[\frac{1}{d} \ln \left(\frac{E / n_{0}}{c}\right)\right]^{2}\right\} .
$$

The values of the four parameters in Eq. (1) are given in Table I, where the large number of significant figures is needed to reproduce the mobility values but are not otherwise meaningful. When $E / n_{0}<c$, Eq. (1) is equivalent to an expansion of $K_{0}$ in powers of $E / n_{0}$, which is known ${ }^{18}$ to be proper at low $E / n_{0}$. However, we found empirically that we could achieve a closer match to the experimental data above $25 \mathrm{Td}\left(1 \mathrm{Td}=10^{-21} \mathrm{~V} \mathrm{~m}^{2}\right)$ by using the same six-parameter formula $^{21}$ that was used in our earlier work; ${ }^{17}$ the six parameters obtained by nonlinear fitting with a generic algorithm are given in Table I. Both the raw and smoothed data in Fig. 1 have been placed into the gaseous ion database. ${ }^{20}$

The results in Fig. 1 show that a clear difference begins to set in at about $35 \mathrm{Td}$ between the present results and those measured previously ${ }^{8}$ in essentially the same apparatus. This difference grows larger than the combined errors bars $(5.0 \%)$
TABLE I. Parameters for best fits of Eq. (1) and the six-parameter formula (Ref. 17) to the data in Fig. 1.

\begin{tabular}{ll}
\hline \hline$K_{0}(0)$ & $8.3343855 \mathrm{~cm}^{2} / \mathrm{V} \mathrm{s}$ \\
$b_{1}$ & $1.675500 \times 10^{-4} \mathrm{Td}^{-2}$ \\
$b_{2}$ & $-1.8125347 \times 10^{-4} \mathrm{Td}^{-4}$ \\
$b_{3}$ & $1.420864 \times 10^{-8} \mathrm{Td}^{-2}$ \\
$a_{1}$ & $-5.420442 \times 10^{-4} \mathrm{Td}^{-2}$ \\
$a_{2}$ & $2.433956 \times 10^{-7} \mathrm{Td}^{-4}$ \\
$b$ & $1.6849085 \mathrm{~cm}^{2} / \mathrm{V} \mathrm{s}$ \\
$c$ & $51.9977478 \mathrm{Td}$ \\
$d$ & 0.58737767 \\
\hline \hline
\end{tabular}

by about $60 \mathrm{Td}$. Based on the considerations given above, the present smoothed results are expected to be accurate within $4 \%$.

\section{INTERACTION POTENTIALS}

The ground state of $\mathrm{O}^{+}$is ${ }^{4} S$, the first excited state $\left({ }^{2} D\right)$ lies about $3 \mathrm{eV}$ higher in energy, and there are no low-lying electronic states of Ne. Therefore, we expect a singlereference $a b$ initio method to perform very well for $\mathrm{NeO}^{+}$. As in our previous work, ${ }^{17}$ we employ the $\operatorname{RCCSD}(\mathrm{T})$ method $^{22}$ in MOLPRO ${ }^{23}$ with the aug-cc-pV5Z basis set. Potential energy curves were calculated point by point, with full counterpoise corrections, across a wide range of internuclear separations $R$, spanning the short-, medium-, and long-range regions. This gives the potential energy curves in the absence of spin-orbit coupling.

Note that all of the RCCSD(T) calculations employed the full counterpoise correction based on $\mathrm{Ne}$ and $\mathrm{O}^{+}$as the fragments, with the latter in its correct atomic state for the two different molecular states. Such a calculation yields the counterpoise-corrected interaction energy relative to the respective asymptotes, and so it was necessary to shift the counterpoise-corrected $\mathrm{RCCSD}(\mathrm{T})$ curves with respect to each other, in order to put them on the same energy scale prior to the spin-orbit interaction. This was done by applying an upward shift of $26818.6 \mathrm{~cm}^{-1}$ to the ${ }^{2} D$ curve, based on the experimental separations of the $\mathrm{O}^{+}{ }^{4} S$ and ${ }^{2} D$ states, with the latter energy being derived from the energies of the ${ }^{2} D_{5 / 2}$ and ${ }^{2} D_{3 / 2}$ levels.

Our experience with oxygen anions ${ }^{13}$ indicates that the effect of the counterpoise correction is small (of the order of $5 \mathrm{~cm}^{-1}$ for $\mathrm{HeO}^{-}$and $13 \mathrm{~cm}^{-1}$ for $\mathrm{NeO}^{-}$). The effect is expected to be even smaller here, owing to the more contracted $\mathrm{O}^{+}$orbitals, and the percentage changes about three times smaller, owing to the increased interaction energy. Thus, with basis sets of the size employed herein, basis set superposition error is not a significant problem.

Spin-orbit coupling was included by employing the Breit-Pauli operator, ${ }^{24}$ as implemented in MOLPRO. ${ }^{23}$ Stateaveraged complete active space self-consistent field (CASSCF) calculations are carried out with the RCCSD(T) energies employed as the unperturbed values for the spinorbit calculations. The CASSCF calculations employed the frozen-core approximation. The standard aug-cc-pVQZ basis sets were employed, including $s, p, d$, and $f$ functions for $\mathrm{Ne}$ and $\mathrm{O}$, all uncontracted. 


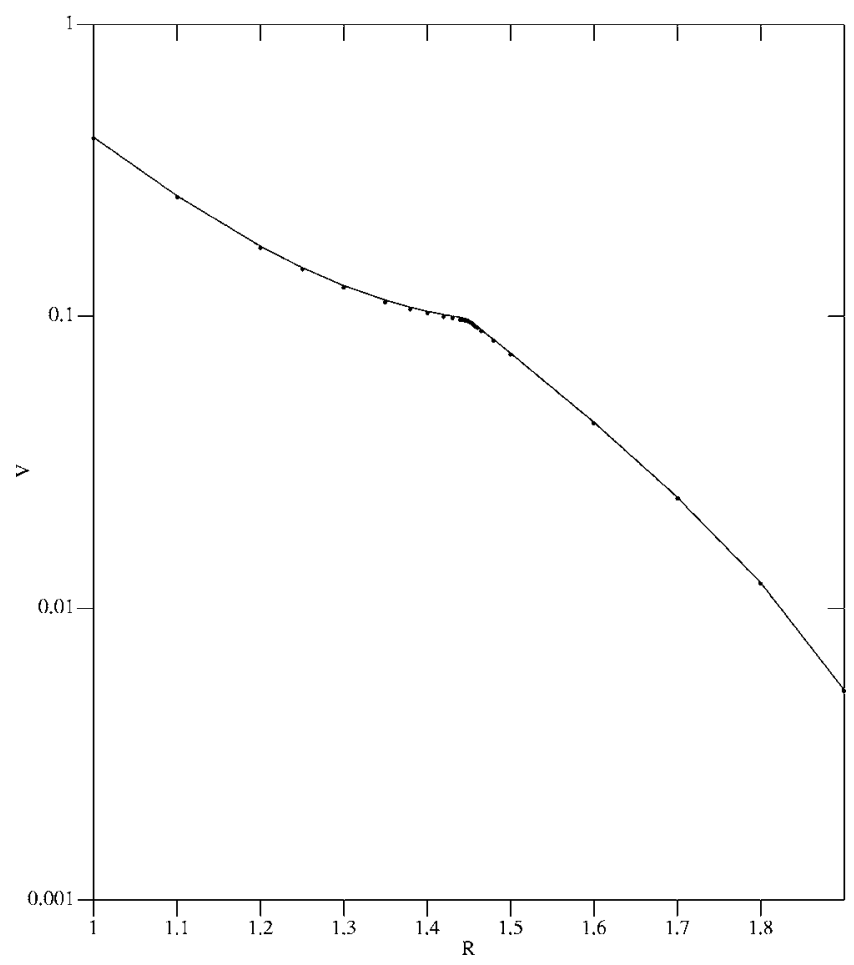

FIG. 2. Interaction potential $V$, in hartree, at short separations $R$, in $\AA$, for $\mathrm{NeO}^{+}$. The dots are the combined potential obtained from the ${ }^{2} \Pi_{3 / 2}$ potential below the avoided crossing and the ${ }^{4} \Sigma_{3 / 2}^{-}$potential above this separation. The curve is the combined potential obtained from the ${ }^{2} \Pi_{1 / 2}$ and ${ }^{4} \Sigma_{1 / 2}^{-}$ potentials.

When spin-orbit coupling is considered, the ${ }^{4} \Sigma^{-}$ground state of the molecular ion has two $\Omega$ components, ${ }^{4} \Sigma_{1 / 2}^{-}$and ${ }^{4} \Sigma_{3 / 2}^{-}$, owing to the different projections of $\mathbf{S}$ on the internuclear axis. These states can then interact with others that have $\Omega=1 / 2$ or $3 / 2$. We initially considered the ${ }^{2} \Pi,{ }^{2} \Delta$, and ${ }^{2} \Sigma^{-}$states, but the ${ }^{2} \Delta$ and ${ }^{2} \Sigma^{-}$are not expected to cross the ${ }^{4} \Sigma^{-}$state, by analogy with $\mathrm{HeO}^{+}$, and hence were ignored (since our focus is on the ground state of the molecular ion). The ${ }^{2} \Pi-{ }^{4} \Sigma^{-}$crossing is on the repulsive wall but not as relatively far up on this wall as it was for $\mathrm{HeO}^{+}{ }^{17}$ In that work, we only considered the avoided crossing between the ${ }^{2} \Pi_{3 / 2}$ and ${ }^{4} \Sigma_{3 / 2}^{-}$states; what this implicitly assumed was that the ${ }^{2} \Pi_{1 / 2}{ }^{4} \Sigma_{1 / 2}^{-}$interacting pair of curves was parallel to the ${ }^{2} \Pi_{3 / 2^{-}}{ }^{4} \Sigma_{3 / 2}^{-}$interacting pair. In the present work on $\mathrm{NeO}^{+}$, we have explicitly considered both pairs of potentials; the differences are indeed very small. Numerical results for all of these spin-orbit potentials over a wide range of separations are available upon request.

We have also recomputed the potentials for $\mathrm{HeO}^{+}$and verified that the differences between the ${ }^{2} \Pi_{1 / 2^{-}}{ }^{4} \Sigma_{1 / 2}^{-}$and ${ }^{2} \Pi_{3 / 2}{ }^{4} \Sigma_{3 / 2}^{-}$interacting pairs are small in that system as well. This is as expected, since the ${ }^{4} \Sigma_{3 / 2}^{-}$and ${ }^{4} \Sigma_{1 / 2}^{-}$curves are degenerate and the ${ }^{2} \Pi_{3 / 2}$ and ${ }^{2} \Pi_{1 / 2}$ curves are parallel in the absence of interaction between like- $\Omega$ states. When the interaction is turned on, the only feature that can change the parallel nature of the curves is the angles at which the like- $\Omega$ curves cross each other; this is a small effect and localized to the repulsive region of the interactions, where the impact on the calculated mobility is small.

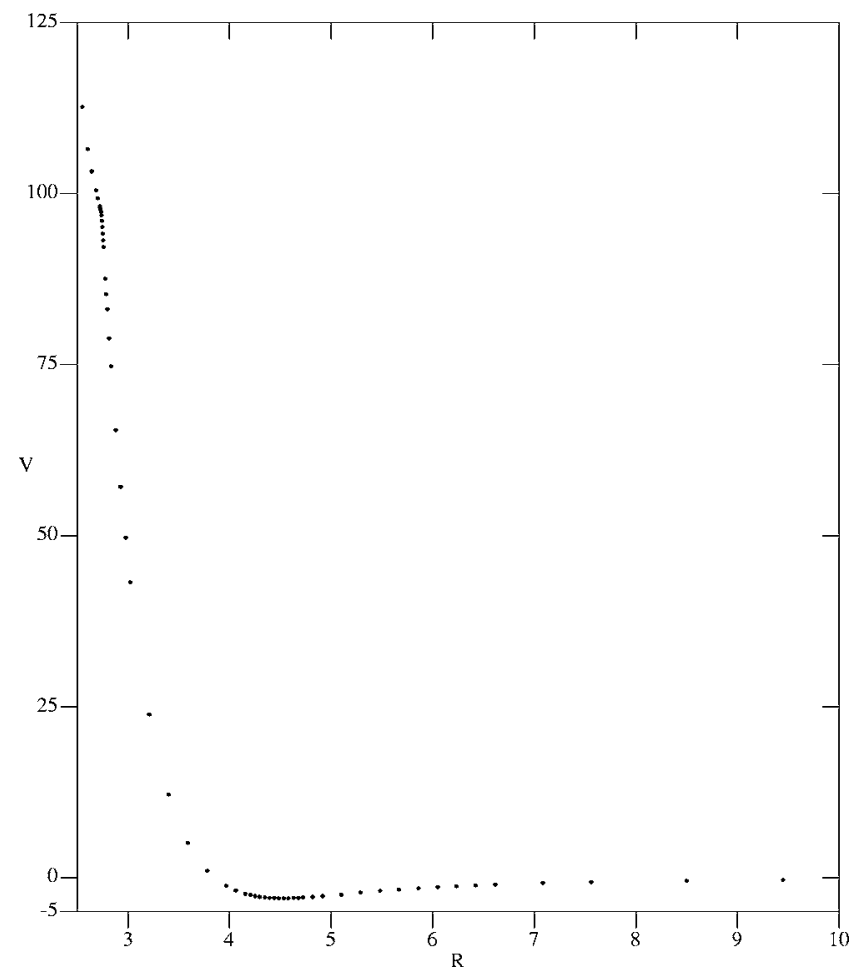

FIG. 3. Interaction potential $V$, in millihartree, as a function of separation $R$, in bohr, for $\mathrm{NeO}^{+}$, as obtained from the ${ }^{2} \Pi_{1 / 2}$ potential below the avoided crossing and the ${ }^{4} \Sigma_{1 / 2}^{-}$potential above this separation. The region at small separations, where there are many closely spaced points and a shoulder, is shown in more detail in Fig. 2.

\section{MOBILITY CALCULATIONS}

To compute the gaseous ion mobilities from our ab initio interaction potentials, we first constructed two "combined" potentials. The first consisted of values of the lowest $\Omega=3 / 2$ potential at short $R$, to a point beyond the avoided crossing at $R=1.46 \AA$, where the spin-orbit interaction was negligible owing to the large separation between the ${ }^{4} \Sigma^{-}$and ${ }^{2} \Pi$ states; thereafter, values of the ${ }^{4} \Sigma^{-}$potential were employed. This procedure was adopted to avoid problems in the convergence of the state-averaged CASSCF calculations at long $R$ and because, at long $R$, more states would need to be included to obtain the correct asymptotic behavior of the spin-orbit curves.

The second combined potential was similarly constructed from values of the lowest $\Omega=1 / 2$ curve and the ${ }^{4} \Sigma^{-}$ potential. The combined potentials are very similar, as can be seen in Fig. 2. They are also smooth, even around the avoided crossing and near the potential minimum, as shown in Fig. 3. The potentials, therefore, presented no problems when used in program QVALUES (Refs. 25 and 26) to determine the transport cross sections ${ }^{18}$ with an accuracy of $0.2 \%$ for collision energies between $10^{-9}$ and 10 hartrees.

It is important to note that a feature of both the present work and our previous work ${ }^{17}$ is that we do not take into account quantum interference (resonance) effects that arise due to the curve crossing of the potentials. ${ }^{27}$ The a priori justification for this is that the curve crossings occur at rather high energies on the repulsive walls, and the experimental transport data available for $\mathrm{HeO}^{+}$and $\mathrm{NeO}^{+}$do not extend to 
TABLE II. Calculated spectroscopic values for $\mathrm{NeO}^{+}\left({ }^{4} \Sigma^{-}\right)$.

\begin{tabular}{cc}
\hline \hline Parameter & Value $\left(\mathrm{cm}^{-1}\right)$ \\
\hline$D_{e}$ & 623.9 \\
$\omega_{e}$ & 131.4 \\
$\omega_{e} x_{e}$ & 8.1 \\
$B_{e}$ & 0.330 \\
$\alpha$ & 0.021 \\
\hline \hline
\end{tabular}

high enough values of $E / n_{0}$ that they will be significantly affected by the curve crossing. The a posteriori reason is that the agreement between the experimental mobilities and those calculated from our combined potentials is excellent for both systems.

The transport cross sections were used in program GC (Ref. 28) [an improved version of program GRAMCHAR (Ref. 29)] to calculate the transport coefficients with a numerical precision of $0.3 \%$ for the ion mobility and $3 \%$ for the ion diffusion coefficients parallel and perpendicular to an electrostatic field. These calculations were performed using the transport cross sections for each combined potential and also by using a 50-50 mixture of the cross sections for the two potentials. The three sets of results are nearly identical, with the differences being too small to be distinguished on the scale of Fig. 1. The full set of results, including diffusion coefficients, has been entered into the gaseous ion transport database. $^{20}$ In Sec. VI we shall discuss only the results shown in Fig. 1 that were obtained with the 50-50 mixture at $300 \mathrm{~K}$, the temperature of the present experimental data.

\section{SPECTROSCOPY}

From the combined $a b$ initio curves, we were able to extract spectroscopic parameters. As in our previous studies, we employed LeRoy's LEVEL program ${ }^{30}$ to obtain rovibrational energy levels, and from these data derived equilibrium values by least-squares fitting the energies of the lowest few levels to standard spectroscopic expressions. For the lowest $\Omega=3 / 2$ and $1 / 2$ curves employed in the present study, the spectroscopic parameters were identical to three decimal places, owing to the parallel nature of the curves close to the minimum. Thus, we report in Table II only the spectroscopic parameters for the ${ }^{4} \Sigma^{-}$state of $\mathrm{NeO}^{+}$.
The interaction potential of $\mathrm{NeO}^{+}$is just under twice as deep as that for $\mathrm{HeO}^{+}$. This deeper potential supports 15 vibrational energy levels at negative values (relative to the dissociation asymptote) of 560.14, 444.89, 345.77, 262.25, 193.48, 138.40, 95.59, 63.42, 40.10, 23.92, 12.81, 5.82, 1.99, 0.37 , and $0.005 \mathrm{~cm}^{-1}$. It is also interesting to note that both $\mathrm{HeO}^{+}$and $\mathrm{NeO}^{+}$have significantly deeper potentials (by about a factor of 3 ) than their anionic counterparts, ${ }^{13}$ indicating the greater repulsive interaction in the latter species, owing to the extra electrons. Of course, both anionic and cationic species have the same charge-induced dipole interaction between the oxygen ion and the rare gas atom, although the higher multipole terms will differ. This means that the $\mathrm{NeO}^{+}$and $\mathrm{NeO}^{-}$potentials will coincide at very large separations, and similarly for $\mathrm{HeO}^{+}$and $\mathrm{HeO}^{-}$.

\section{DISCUSSION AND CONCLUDING REMARKS}

We have reported new experimental values for the mobility of ground state $\mathrm{O}^{+}$ions in $\mathrm{Ne}$ gas at $300 \mathrm{~K}$. Corrections to the raw data were made to account for the new source of experimental error detected in the Pittsburgh SIDA-nonuniformity of the electric fields in the first $2.5 \mathrm{~cm}$ of the main drift region. The corrected results, shown in Fig. 1 and represented by the parameters given in Table I, are expected to be accurate within $4 \%$.

We have also calculated the mobility of $\mathrm{O}^{+}$in $\mathrm{Ne}$ theoretically, starting from an ab initio interaction potential. Figure 1 shows that the values calculated $a b$ initio lie above the present experimental values but by an amount that is within the experimental error bars indicated on the data point at $65 \mathrm{Td}$. A quantitative measure of this good agreement is given by the values of $\delta$ and $\chi$ shown in Table III. Here "Acc" is the maximum inaccuracy estimated for the experimental data, "Prec" is the maximum imprecision estimated for the theoretical calculations (assuming the interaction potential is correct), and $N$ is the number of experimental data points. Values above 1 or below -1 for the statistical quantity $\delta$ indicate that the experimental values are significantly above or below the calculated values, respectively. Hence the values for $\delta$ in Table III indicate that the present experimental values lie below the calculated ones, but that the

TABLE III. Statistical comparison of experimental mobilities at $300 \mathrm{~K}$ and calculated values.

\begin{tabular}{lcccccc}
\hline \hline Data & $\begin{array}{c}\text { Acc } \\
(\%)\end{array}$ & $\begin{array}{c}\text { Prec } \\
(\%)\end{array}$ & $\begin{array}{c}E / n_{0} \\
(\mathrm{Td})\end{array}$ & $N$ & $\delta$ & $\chi$ \\
\hline Present (raw) & 4 & 0.3 & $3.8-20$ & 51 & -0.94 & 1.05 \\
& 4 & 0.3 & $20-68$ & 48 & -0.98 & 1.04 \\
& 4 & 0.3 & $3.8-68$ & 99 & -0.96 & 1.04 \\
\multirow{2}{*}{ Present (mixed fit) } & 4 & 0.3 & $5.3-20$ & 14 & -0.85 & 0.90 \\
& 4 & 0.3 & $20-68$ & 47 & -0.82 & 0.88 \\
& 4 & 0.3 & $5.3-68$ & 61 & -0.83 & 0.88 \\
Previous $^{\mathrm{a}}$ & & & & & & \\
& 3 & 0.3 & $5.3-20$ & 6 & -0.63 & 0.73 \\
& 3 & 0.3 & $20-60$ & 6 & -2.00 & 2.05 \\
& 3 & 0.3 & $5.0-60$ & 12 & -1.32 & 1.54 \\
\hline \hline
\end{tabular}

${ }^{\mathrm{a}}$ Reference 8 . 
difference never becomes statistically significant. The statistical quantity $\chi$ is always positive, with values not much greater than $|\delta|$, as in Table III, indicating that there is little scatter in the data and that the comparison is nearly independent of $E / n_{0}$. More information about $\delta$ and $\chi$ is given elsewhere. $^{26}$

Table III also compares our calculated mobilities at $300 \mathrm{~K}$ with those measured in $1982 .{ }^{8}$ These older data agree with the present data within the combined experimental uncertainties, but above $20 \mathrm{Td}$ they are in significant disagreement with the calculated values. Since the present experimental values are in agreement with the calculated values at all $E / n_{0}$, and since the agreement does not change with $E / n_{0}$, we conclude that the earlier data above $20 \mathrm{Td}$ were not as accurate as was claimed. The calculated values match the new experimental values within the $4 \%$ accuracy estimated for the latter. This is in sharp contrast with the previous experimental results, which disagree significantly at $E / n_{0}$ values above $35 \mathrm{Td}$ with the calculated values.

In conclusion, the new interaction potentials for $\mathrm{NeO}^{+}$ and the new experimental mobilities for $\mathrm{O}^{+}$in $\mathrm{Ne}$ reported in this paper appear to be the only and most accurate ones available, respectively. Good agreement has been obtained between the mobilities calculated from the new potentials and the new experimental values. Finally, we have calculated the diffusion coefficients of $\mathrm{O}^{+}$ions in $\mathrm{Ne}$ gas at various temperatures, as a function of $E / n_{0}$, and the standard spectroscopic parameters for the $\mathrm{NeO}^{+}$system; unfortunately, no experimental results appear to be available for comparison.

\section{ACKNOWLEDGMENTS}

This material is based on the work supported by the U.S. National Science Foundation under Grant No. CHE0414241. The authors are grateful to the EPSRC for the award of computer time at the Rutherford Appleton Laboratories under the auspices of the Computational Chemistry Working Party.
${ }^{1}$ R. Johnsen, H. L. Brown, and M. A. Biondi, J. Chem. Phys. 52, 5080 (1970).

${ }^{2}$ M. McFarland, D. L. Albritton, F. C. Fehsenfeld, E. E. Ferguson, and A. L. Schmeltekopf, J. Chem. Phys. 59, 6610 (1973).

${ }^{3}$ W. Lindinger and D. L. Albritton, J. Chem. Phys. 62, 3517 (1975).

${ }^{4}$ R. G. Kosmider and J. B. Hasted, J. Phys. B 8, 273 (1975).

${ }^{5}$ I. Dotan, W. Lindinger, and D. L. Albritton, J. Chem. Phys. 64, 4544 (1976).

${ }^{6}$ B. R. Rowe, D. W. Fahey, F. C. Fehsenfeld, and D. L. Albritton, J. Chem. Phys. 73, 194 (1980).

${ }^{7}$ H. A. Fhadil, D. Mathur, and J. B. Hasted, J. Phys. B 15, 1443 (1982).

${ }^{8}$ R. Johnsen, M. A. Biondi, and M. Hayashi, J. Chem. Phys. 77, 2545 (1982).

${ }^{9}$ M. H. Khatri, J. Phys. D 17, 273 (1984).

${ }^{10}$ D. L. Albritton, I. Dotan, W. Lindinger, M. McFarland, J. Tellinghuisen, and F. C. Fehsenfeld, J. Chem. Phys. 66, 410 (1977).

${ }^{11}$ L. A. Viehland and E. A. Mason, J. Chem. Phys. 66, 422 (1977)

${ }^{12}$ S. L. Lin and J. N. Bardsley, J. Chem. Phys. 66, 435 (1977).

${ }^{13}$ L. A. Viehland, R. Webb, E. P. F. Lee, and T. G. Wright, J. Chem. Phys. 122, 114302 (2005).

${ }^{14}$ T. G. Wright and L. A. Viehland, Chem. Phys. Lett. 420, 24 (2006).

${ }^{15}$ E. Qing, L. A. Viehland, E. P. F. Lee, and T. G. Wright, J. Chem. Phys. 124, 044316 (2006).

${ }^{16}$ B. R. Gray, E. P. F. Lee, A. Yousef, S. Shrestha, L. A. Viehland, and T. G. Wright, Mol. Phys. 104, 3237 (2006).

${ }^{17}$ D. M. Danailov, R. Brothers, L. A. Viehland, R. Johnsen, T. G. Wright, and E. F. P. Lee, J. Chem. Phys. 125, 084309 (2006).

${ }^{18}$ E. A. Mason and E. W. McDaniel, Transport Properties of Ions in Gases (Wiley, New York, 1988).

${ }^{19}$ H. Helm and M. T. Elford, J. Phys. B 10, 983 (1977).

${ }^{20}$ To access this database you must telnet to the computer named sassafrass.chatham.edu and login as gastrans. The required password will be provided upon request by email to viehland@sassafrass.chatham.edu

${ }^{21}$ M. Saporoschenko, Phys. Rev. A 8, 1044 (1973).

${ }^{22}$ C. Hampel, K. Peterson, and H.-J. Werner, Chem. Phys. Lett. 190, 1 (1992).

${ }^{23}$ H.-J. Werner, P. J. Knowles, J. Almlof et al., MOLPRO, a package of $a b$ initio programs

${ }^{24}$ A. Berning, M. Schweizer, H.-J. Werner, P. J. Knowles, and P. Palmiere, Mol. Phys. 98, 1823 (2000).

${ }^{25}$ L. A. Viehland, Chem. Phys. 78, 149 (1982).

${ }^{26}$ L. A. Viehland, Chem. Phys. 85, 291 (1984).

${ }^{27}$ R. E. Olson, Phys. Rev. A 2, 121 (1970).

${ }^{28}$ A. Yousef, S. Shrestha, L. A. Viehland, B. R. Gray, and T. G. Wright, J. Chem. Phys. (to be published).

${ }^{29}$ L. A. Viehland, Chem. Phys. 179, 71 (1994).

${ }^{30}$ R. J. LeRoy, "LEVEL 7.2: A Computer Program for Solving the Radial Schrödinger Equation for Bound and Quasibound Levels, and Calculating Various Values and Matrix Elements," University of Waterloo Chemical Physics Research Program Report No. CP-555R, 2000 (unpublished). 